Journal for ImmunoTherapy of Cancer mutation burden for predicting immune checkpoint blockade response: the more, the better. Journal for ImmunoTherapy of Cancer 2022;10:e003087. doi:10.1136/jitc-2021-003087

- Additional supplemental material is published online only. To view, please visit the journal online (http://dx.doi.org/10. 1136/jitc-2021-003087).

Accepted 25 October 2021

Check for updates

(C) Author(s) (or their employer(s)) 2022. Re-use permitted under CC BY-NC. No commercial re-use. See rights and permissions. Published by BMJ.

${ }^{1}$ Institute of Military Cognition and Brain Sciences, Academy of Military Medical Sciences, Beijing, China

${ }^{2}$ Beijing Institute of Basic Medical Sciences, Beijing, China

Correspondence to Dr Ming Zheng; mmzheng@fmmu.edu.cn; zhengming_china@163.com

\title{
Tumor mutation burden for predicting immune checkpoint blockade response: the more, the better
}

\author{
Ming Zheng (D) ${ }^{1,2}$
}

\section{ABSTRACT}

Background Recently, the US Food and Drug Administration (FDA) has approved immune checkpoint blockade (ICB) for treating cancer patients with tumor mutation burden (TMB) $>10$ mutations/megabase (mut/ $\mathrm{Mb})$. However, high TMB (TMB-H) defined by $>10$ mut/ $\mathrm{Mb}$ fails to predict ICB response across different cancer types, which has raised serious concerns on the current FDA approval. Thus, to better implement TMB as a robust biomarker of ICB response, an optimal and generalizable TMB cut-off within and across cancer types must be addressed as soon as possible.

Methods Using Morris's and Kurzrock's cohorts ( $n=1662$ and 102), we exhaustively tested all possible TMB cut-offs for predicting ICB treatment outcomes in 10 cancer types. The bootstrap method was applied to generate 10,000 randomly resampled cohorts using original cohorts to measure the reproducibility of TMB cut-off. ICB treatment outcomes were analyzed by overall survival, progression-free survival and objective response rate.

Results No universally valid TMB cut-off was available for all cancer types. Only in cancer types with higher TMB (category I), such as melanoma, colorectal cancer, bladder cancer, and non-small cell lung cancer, the associations between TMB-H and ICB treatment outcomes were less affected by TMB cut-off selection. Moreover, high TMB (category I) cancer types shared a wide range of TMB cut-offs and a universally optimal TMB cut-off of 13 mut/Mb for predicting favorable ICB outcomes. In contrast, low TMB (category II) cancer types, for which the prognostic associations were sensitive to TMB cut-off selection, showed markedly limited and distinct ranges of significantly favorable TMB cut-offs. Equivalent results were obtained in the analyses of pooled tumors.

Conclusions Our finding - the correlation that TMB-H is more robustly associated with favorable ICB treatment outcomes in cancer types with higher TMBs - can be used to predict whether TMB could be a robust predictive biomarker in cancer types for which TMB data are available, but ICB treatment has not been investigated. This theory was tested in cancer of unknown primary successfully. Additionally, the universal TMB cut-off of 13 mut/Mb might reveal a general requirement to trigger the sequential cascade from somatic mutations to an effective antitumor immunity.

\section{BACKGROUND}

The immune system possesses the potential ability to fight cancer, but its anticancer ability is held in check by immune checkpoints. Immune checkpoint blockade (ICB) therapy, such as anti-CTLA-4, anti-PD-1, and anti-PD-L1, can unleash anticancer immunity and mediate durable tumor regression in many cancers. ${ }^{1}$ Such successes have led to the clinical development and approval of ICB treatment as a promising therapy for patients with cancer. However, even in ICBresponsive cancer types, only a small subset of patients with cancer have robust and durable responses to ICB treatment. Durable objective responses to ICB treatment were achieved in only $31 \%-44 \%$ of advanced melanoma, $22 \%-25 \%$ of renal cell carcinoma, and $19 \%-20 \%$ of non-small-cell lung cancer patients. ${ }^{2}$ Thus, ICB treatment benefits some patients, but predictive biomarkers are needed for more precise therapy.

Recently, tumor mutation burden (TMB) has emerged as a predictive biomarker of ICB response in diverse cancers. ${ }^{3}$ With the advent of TMB quantification techniques by nextgeneration sequencing (NGS) and interrogation of hundreds of patients, a significant but not absolute relationship between high TMB (TMB-H) and improved responsiveness to ICB treatment has been established. ${ }^{45}$ Recently, the US Food and Drug Administration (FDA) approved pembrolizumab (anti-PD-1) for the treatment of cancer patients with TMB $>10$ mutations/megabase (mut/Mb). ${ }^{6}$ Although the approval provides a novel therapeutic option for cancer patients, it raises major concerns: (1) a TMB of 10 mut/Mb is an arbitrary and capricious cut-off for ICB treatment selection ${ }^{7}$; and (2) TMB-H defined by $>10$ mut/Mb fails to predict improved ICB response across different cancer types. ${ }^{8}$ Therefore, the reproducibility of TMB cut-off has become the focal point for ICB treatment. To better implement TMB as a robust biomarker for ICB response, an optimal and generalizable TMB cut-off across different cancer types must be addressed as soon as possible. 


\section{METHODS}

\section{Patients with cancer treated with ICB}

Two cohorts of ICB-treated cancer patients were acquired from previous studies. ${ }^{45}$ Morris's cohort included 1662 patients with cancer treated with ICB whose tumors were analyzed by NGS to measure TMB. The patients' TMB values and detailed clinical information, including sex, age, drug class and year of ICB start, were retained from the previous study. ${ }^{5}$ The Morris's ICB-treated cancer patients were used for overall survival (OS) analysis. Kurzrock's ICB cohort included 151 immunotherapy-treated patients with cancer, ${ }^{4}$ and 102 ICB-treated patients who underwent TMB assessment were used for analyzing progression-free survival (PFS) and ICB response rate.

\section{An exhaustive survival analysis framework to evaluate the robustness of prognostic relevance}

We conducted an exhaustive survival analysis framework using the survivALL R package (https://CRAN.R-project. org/package=survivALL).${ }^{9} 10$ To analyze the prognostic relevance of a continuous TMB gradient, all possible TMB cut-offs were independently tested for predicting the prognosis of patients with cancer. According to each TMB cut-off, patients were separated into TMB-H and low TMB (TMB-L) groups to compare survival following ICB treatment (TMB-H vs TMB-L). Next, the robustness of prognostic relevance was measured by the percentages of significantly favorable and unfavorable TMB cut-offs. The hazard ratios (HRs) were calculated using all significant cutoffs. Additionally, the bootstrap resampling method ${ }^{11} 12$ was applied to generate 10,000 randomly resampled cohorts with replacement from the original cohort. Next, statistical estimates, including the mean, standard error (SE), and 95\% confidence interval (CI), were calculated by 10,000 bootstrap replicates. The bootstrap resampling analysis was conducted using the sample function of the base package in R (The R Foundation for Statistical Computing, Vienna, Austria, http:/ /www.r-project.org).

\section{Statistical analysis}

All statistical analyses were performed by using R V.3.6.1. Student's t-test, $\chi^{2}$ test, and Spearman's correlation were conducted using the stats $\mathrm{R}$ package. ${ }^{13}$ The $\chi^{2}$ test was based on 10,000 replicates by Monte Carlo simulation. ${ }^{14}$ All statistical tests used 0.05 as the significance level, $\mathrm{p} \geq 0.05$ was considered not significant (ns), and $\mathrm{p}<0.05$ was considered a statistically significant difference, indicated with asterisks $\left({ }^{*} \mathrm{p}<0.05,{ }^{*} \mathrm{p}<0.01, * * * \mathrm{p}<0.001\right.$ and $* * * * \mathrm{p}<0.0001)$.

\section{RESULTS}

TMB-H is more robustly associated with improved OS following ICB treatment in cancer types with higher TMB

The prevalence of TMB among cancers ranges from 0.01 $\mathrm{mut} / \mathrm{Mb}$ to more than $400 \mathrm{mut} / \mathrm{Mb}$. In the wide range of TMB, the arbitrary and capricious selection of TMB cutoffs has limited its clinical application. ${ }^{15}$ Here, we used an exhaustive survival analysis framework, as previously reported, ${ }^{910}$ to analyze all possible TMB cut-offs for evaluating their prognostic associations (figure 1A). According to each TMB cut-off, patients were separated into TMB-H and TMB-L groups to compare the OS following ICB treatment. Based on this analysis, we calculated the percentages of significantly favorable and unfavorable TMB cut-offs, which measure the robustness of prognostic association, indicating whether the prognostic association is affected by different selections of TMB cut-offs. Prognostic value was calculated using HRs of all significant cut-offs (figure 1A). In the analysis, we applied the bootstrap resampling method, a commonly used approach for measuring uncertainty and variability in estimated statistics, ${ }^{11}{ }^{12}$ to generate 10,000 randomly resampled cohorts using the original cohort. The resampled cohorts were used to estimate the reproducibility of the association between TMB and OS following ICB.

Through conducting the exhaustive survival analysis on the Morris's ICB cohort of 1662 cancer patients, we investigated whether TMB-H was associated with improved OS following ICB treatment. We found that, except for breast cancer and glioma, TMB-H was clearly associated with favorable OS in most cancer types (figure 1B). However, it is worth noting that different cancer types showed remarkably distinct robustness of prognostic relevance (figure 1B).

Since TMB varies dramatically across cancer types, ${ }^{16} 17$ we hypothesized that TMB-H might better predict ICBtreated patient survival in cancer types with higher TMB. Thus, we analyzed the correlation of the median TMB values in different cancer types with the robustness of prognostic associations and the prognostic values. The median TMB values were positively associated with the proportions of significantly favorable TMB cut-offs (univariate analysis: Spearman's $\mathrm{rho}=0.824, \mathrm{p}=0.006$; multivariate analysis: Spearman's rho $=0.765, \mathrm{p}=0.016$; figure 1C). Next, according to the TMB levels, we separated cancer types into two categories: (1) cancer types with high TMB (category I): melanoma, colorectal cancer, bladder cancer, and non-small cell lung cancer and (2) cancer types with low TMB (category II): oesophagogastric cancer, head and neck cancer, glioma, breast cancer, and renal cell carcinoma. Compared with low TMB (category II) cancer types, high TMB (category I) cancer types showed significantly higher proportions of significantly favorable TMB cut-offs (figure 1D), indicating higher robustness of favorable prognostic association.

In contrast, TMB-H exhibited minimal associations with unfavorable OS following ICB (figure 1E-F). Notably, there was no significant association between TMB-H and unfavorable OS in high TMB (category I) cancer types (figure 1F). Moreover, cancer types with higher TMB showed a slightly insignificant trend toward lower negative log2-HR, indicating improved OS in patients with TMB-H tumors than in those with TMB-L tumors (figure 1G-H).

Herein, TMB-H/TMB-L means individual patients with tumors of high or low TMB status defined by a specific TMB cut-off. Additionally, high/low TMB (category I/II) 
A
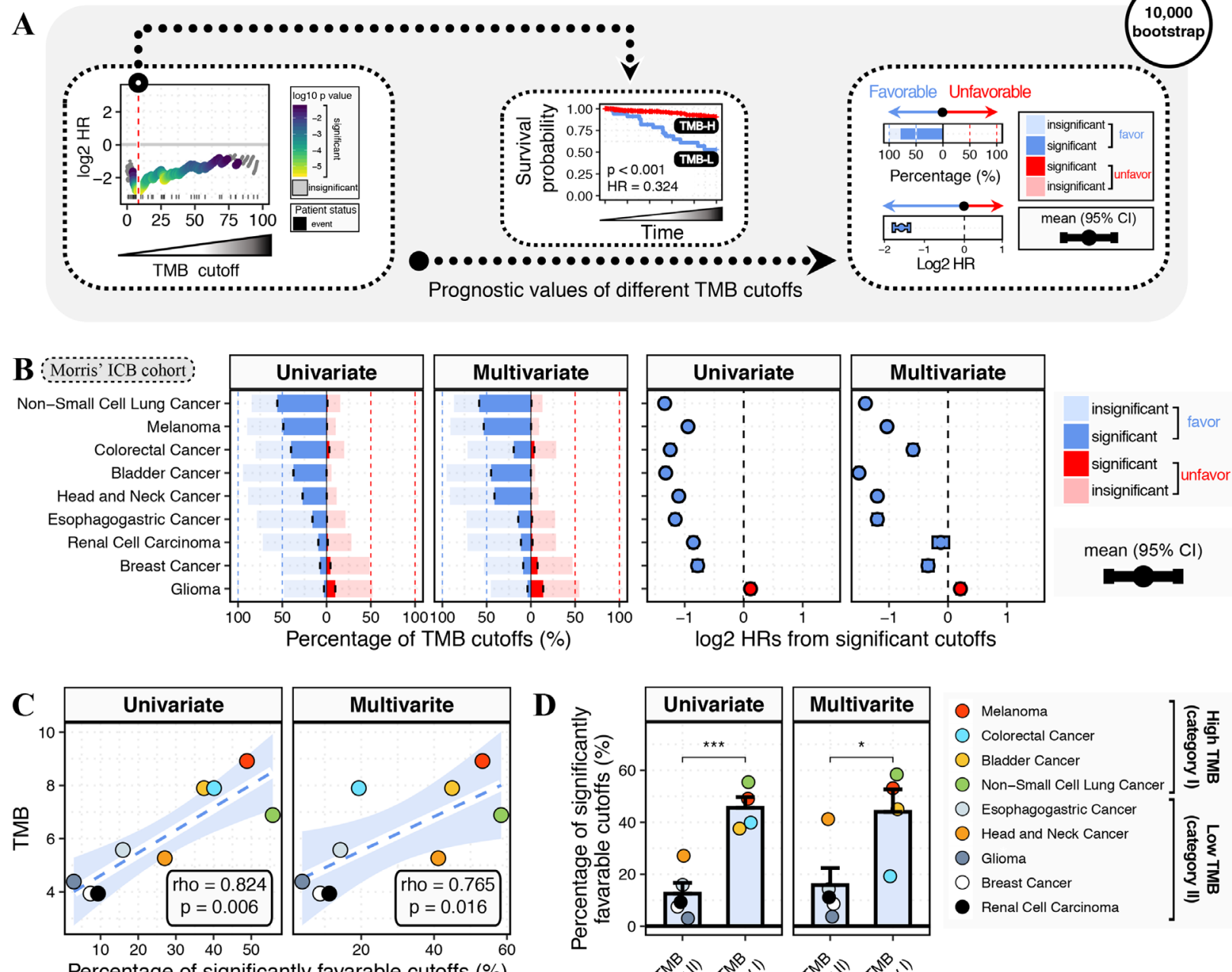

Percentage of significantly favarable cutoffs (\%)
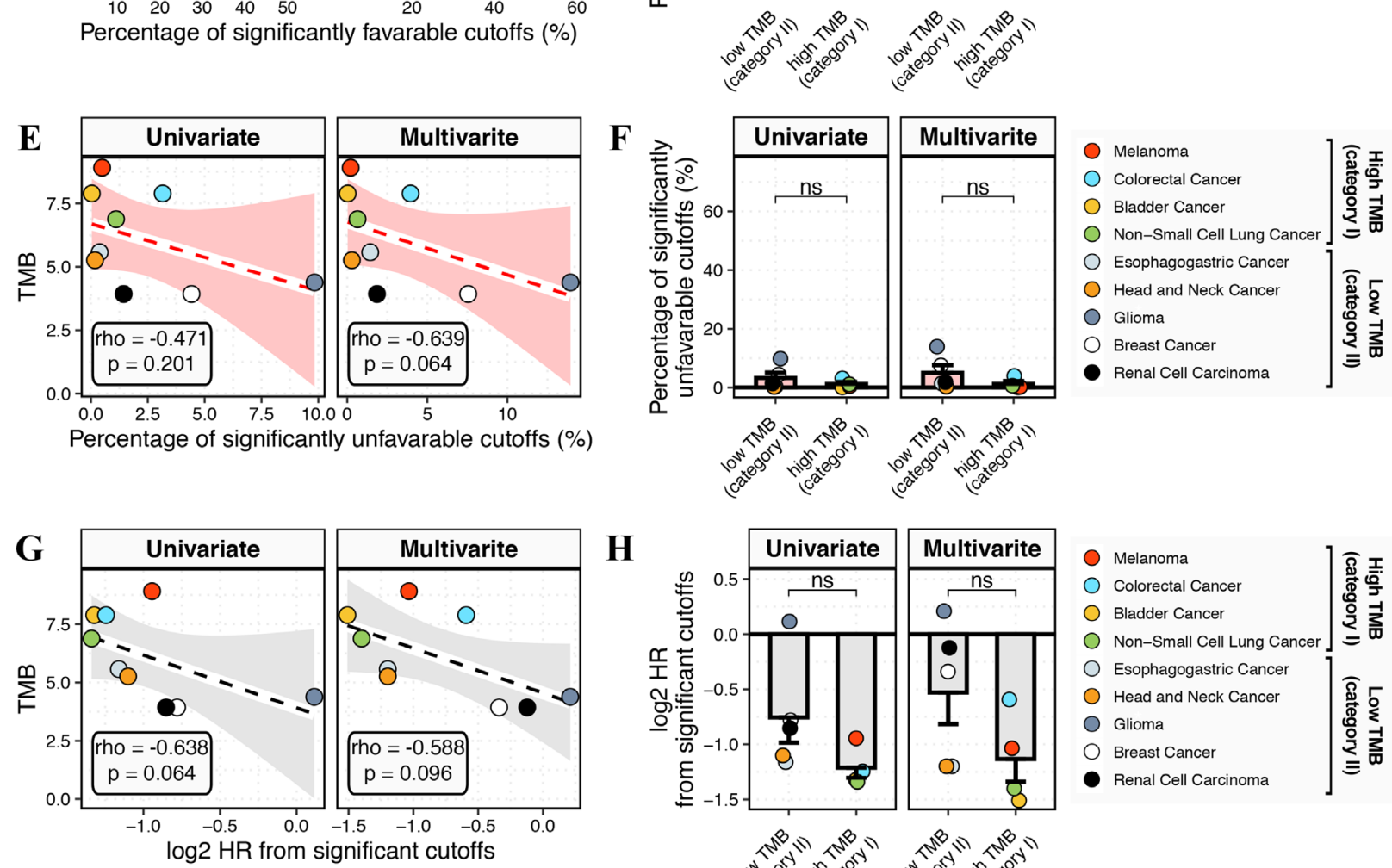

$\mathbf{H}$
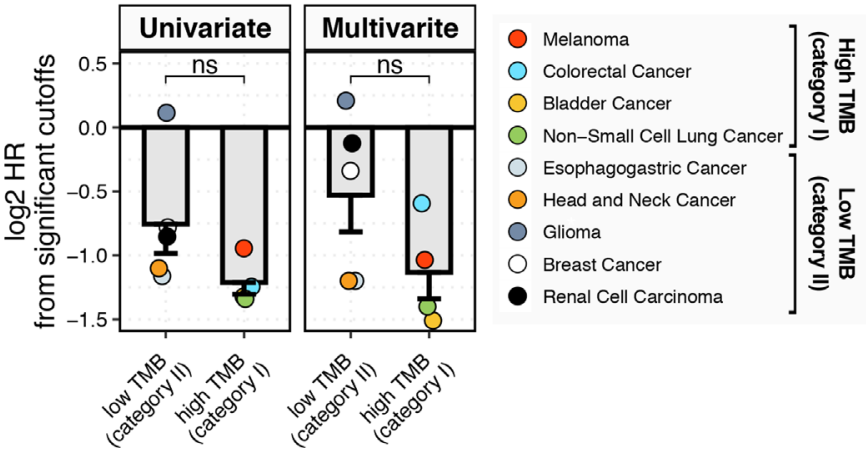

Figure 1 (Continued) 
Figure 1 High tumor mutation burden (TMB-H) is more robustly associated with improved overall survival (OS) following immune checkpoint blockade (ICB) treatment in cancer types with higher TMB. (A) Diagram of exhaustive survival analysis. Using all the possible TMB cut-offs, patients were separated into high TMB (TMB-H) and low TMB (TMB-L) groups to compare OS following ICB treatment (TMB-H vs TMB-L). The robustness of prognostic relevance was measured by the percentages of significantly favorable and unfavorable TMB cut-offs. Prognostic values were calculated by the HRs of all significant cutoffs. In this analysis, the bootstrap resampling method was applied to generate 10,000 randomly resampled cohorts using the original cohort, which were used to estimate the reproducibility of the prognostic association. (B) The relationships between TMB and OS across different cancer types in the Morris's ICB cohort. Both univariate and multivariate Cox proportional hazards regression analyses were conducted to evaluate the OS of ICB-treated patients. Multivariate analysis was performed with covariates of sex, age, drug class, and year of ICB start. Bar plots show the percentages of favorable and unfavorable TMB cut-offs, with error bars representing the SE calculated by 10,000 bootstrap replicates (left plot). Dot plots show the average HR calculated using all significant TMB cut-offs, with error bars representing the mean $\pm 95 \% \mathrm{Cl}$ calculated by 10,000 bootstrap replicates (right plot). The negative log2-HR represents a better prognosis in patients with TMB-H tumors. Different colors correspond to favorable and unfavorable associations, with the color gradient showing the prognostic significance. (C-D) TMB-H is associated with favorable OS following ICB treatment in cancer types with higher TMB. (C) The correlation between the median TMB values and the percentages of significantly favorable TMB cut-offs in different cancer types. Colors correspond to cancer types. Spearman's rho coefficient with $\mathrm{p}$ value and the linear regression line with $95 \% \mathrm{Cl}$ (dashed line and shade) are indicated in each graph. (D) The percentages of significantly favorable TMB cut-offs in two categories of cancer types defined by TMB levels. High TMB (category I) cancer types: melanoma, colorectal cancer, bladder cancer, and non-small cell lung cancer; low TMB (category II) cancer types: oesophagogastric cancer, head and neck cancer, glioma, breast cancer, and renal cell carcinoma. Error bars show the mean \pm SE. The results were considered statistically significant when $p<0.05\left({ }^{*}\right), p<0.01$ $\left.\left(^{\star \star}\right), p<0.001{ }^{\star \star \star}\right)$, and $\left.p<0.0001{ }^{* \star \star \star}\right)$ and insignificant when $p \geq 0.05$ (ns) using Student's t-test. (E-F) TMB-H is not associated with unfavorable OS following ICB treatment. (E) The correlation between the median TMB values and the percentages of significantly unfavorable TMB cut-offs in different cancer types. $(F)$ The percentages of significantly unfavorable TMB cut-offs in high TMB (category I) and low TMB (category II) cancer types. (G-H) TMB-H is associated with improved ICB treatment outcome. (G) The correlation between the median TMB values and the average HRs of significant TMB cut-offs in different cancer types. (H) The average HRs of significant TMB cut-offs in high TMB (category I) and low TMB (category II) cancer types.

cancer type means a cancer type with a relatively higher or lower median TMB score.

\section{TMB-H is associated with improved PFS following ICB treatment in high TMB (category I) cancer types}

Next, we sought to evaluate the ability of TMB-H to predict the PFS of ICB-treated patients in high TMB (category I) cancer types. In Kurzrock's ICB cohort, ${ }^{4}$ two cancer types-melanoma and non-small cell lung cancer (NSCLC) -include more than 35 patients for further analysis. Additionally, melanoma and NSCLC had the highest and lowest TMB levels among the high TMB (category I) cancer types, respectively. Here, we found that TMB-H was remarkably associated with improved PFS after ICB treatment in melanoma and NSCLC (figure 2A). Additionally,

B Morris vs. Kurzrock's ICB cohort:

\section{A Kurzrock's ICB cohort:}
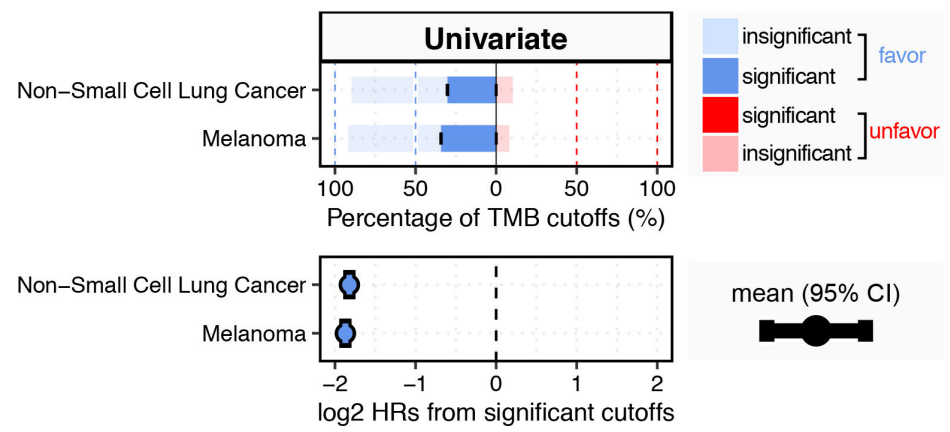

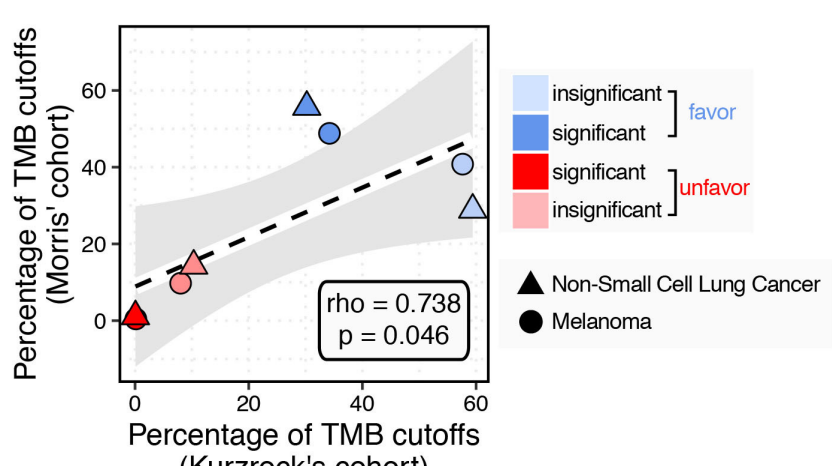

(Kurzrock's cohort)

Figure 2 TMB-H is associated with improved progression-free survival (PFS) following ICB treatment in high TMB (category I) cancer types. (A) The prognostic relevance of TMB in melanoma and non-small cell lung cancer (NSCLC) from Kurzrock's ICB cohort. Univariate Cox proportional hazards regression analyses were conducted to evaluate the PFS of ICB-treated patients. Bar plots show the percentages of favorable and unfavorable TMB cut-offs, with error bars representing the SE calculated by 10,000 bootstrap replicates (upper plot). Dot plots show the average HR of significant TMB cut-offs, with error bars representing the mean $\pm 95 \% \mathrm{Cl}$ calculated by 10,000 bootstrap replicates (lower plot). Different colors correspond to the significance of favorable and unfavorable associations, with the color gradient showing the prognostic significance. (B) The prognostic values of TMB in melanoma and NSCLC from Morris's and Kurzrock's ICB cohorts. Dot colors correspond to the significance of favorable and unfavorable associations. Dot shapes represent cancer types. Spearman's rho coefficient with $\mathrm{p}$ value and the linear regression line with $95 \% \mathrm{Cl}$ (gray dashed line and shade) are indicated in the graph. ICB, immune checkpoint blockade; TMB, tumor mutation burden; TMB-H, high TMB. 
A Morris ICB colort:
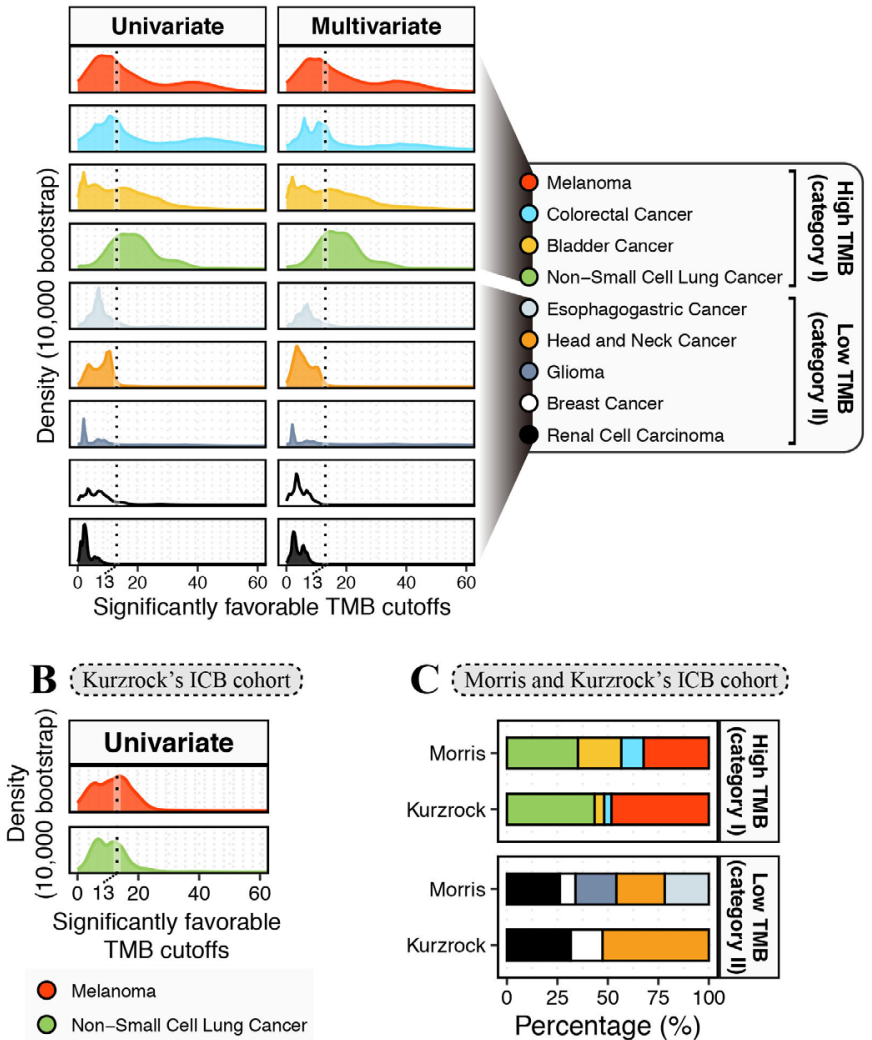

D Morris ICB cohort:

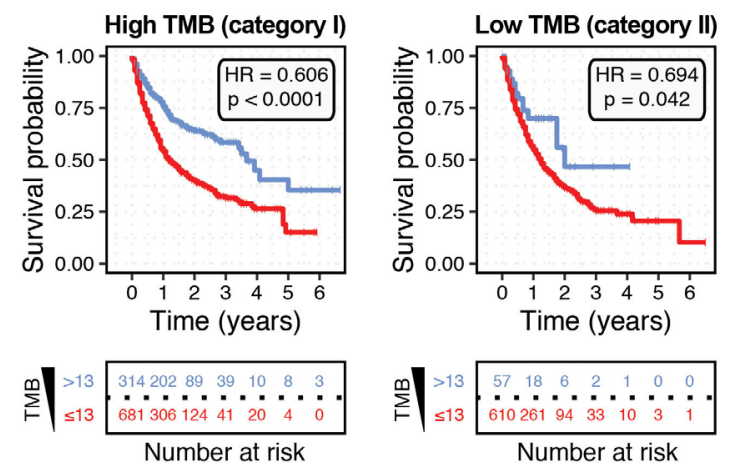

E Kurzock's ICB cohort:
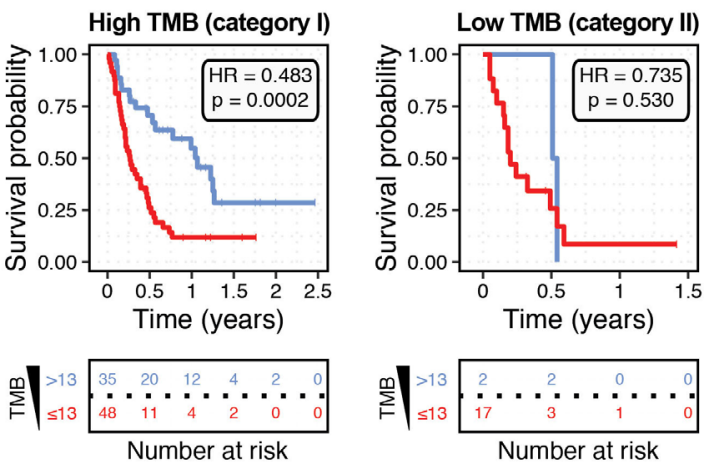

Figure 3 Remarkable shifts in the distributions of significant TMB cut-offs for predicting favorable ICB treatment outcomes across different cancer types and the universal TMB cut-off in high TMB (category I) cancer types. (A-B) The shift in the distributions of significant TMB cut-offs for predicting favorable ICB treatment outcomes across different cancer types. Density plots show the distributions of significantly favorable TMB cut-offs calculated by 10,000 bootstrap replicates. Different colors represent cancer types in both Morris's (A) and Kurzrock's (B) cohorts. The black dashed line indicates the universal TMB cut-off of $13 \mathrm{mut} / \mathrm{Mb}$ in high TMB (category I) cancer types. (C) The percentages of different cancer types with high TMB (category I) and low TMB (category II) in Morris's and Kurzrock's cohorts, with color coding according to cancer types as in figure 3A. (D-E) Kaplan-Meier curves show the survival of ICB-treated patients in Morris's (D) and Kurzrock's (E) cohorts. Patients were divided into two subsets of high TMB (category I; left plot) and low TMB (category II; right plot) tumors. The log-rank $p$ value and HR show the comparison between patients with TMB (mut/Mb) $\leq 13$ and $>13$. The bottom panel shows the number of patients at risk every year. ICB, immune checkpoint blockade; mut/Mb, mutations/megabase; TMB, tumor mutation burden.

the robustness of prognostic associations between TMB-H and improved ICB treatment outcomes were comparable between Morris's and Kurzrock's ICB cohorts (figure 2B).

\section{Remarkable shifts in the distributions of significant TMB cut- offs for predicting favorable ICB treatment outcomes across different cancer types and the universal TMB cut-off in high TMB (category I) cancer types}

It has been reported that the FDA-approved TMB-H $(>10$ mut $/ \mathrm{Mb}$ ) fails to predict ICB response across different cancer types. ${ }^{8}$ Thus, a robust TMB cut-off must be addressed to implement TMB as a reliable clinical biomarker. However, different cancer types had markedly varying TMB levels and, as shown in figure $1 \mathrm{~B}$, the prognostic association between TMB-H and ICB response was disproportionately affected by different TMB cut-offs. Next, to explore the optimal TMB cut-off, we constructed the distributions of significant TMB cut-offs to predict favorable ICB treatment outcomes, which were calculated by 10,000 bootstrap replicates as described in figure 1A. The density plots in figure $3 \mathrm{~A}$ show remarkable shifts in the distributions of significantly favorable TMB cutoffs across different cancer types, indicating that no universal TMB cut-off could be found across all cancer types. Next, the shapes of the distributions were analyzed by the full width at half maximum (FWHM) (online supplemental figure S1A-B). The distributions of significantly favorable TMB cutoffs in high TMB (category I) cancer types were significantly broader than those in low TMB (category II) cancer types (online supplemental figure S1C). Moreover, we observed that the probability of significantly favorable TMB cut-offs peaked around $13 \mathrm{mut} / \mathrm{Mb}$ among all high TMB (category I) cancer types, as indicated by the black dashed line in figure $3 \mathrm{~A}$. Thus, $13 \mathrm{mut} / \mathrm{Mb}$ was hereafter defined as the universal TMB cut-off for high TMB (category I) cancer types. Moreover, $13 \mathrm{mut} / \mathrm{Mb}$ was also the optimal cut-off for high TMB (category I) cancer types in the Kurzrock's cohort (figure 3B).

The above analyses discovered and validated the universally optimal TMB cut-off of 13 mut/Mb in individual cancer types 
independently. Next, we tested the universal TMB cut-off of $13 \mathrm{mut} / \mathrm{Mb}$ in pooled tumors of diverse cancer types. The patients were pooled into two categories according to cancer types with high TMB (category I) or low TMB (category II) in Morris's and Kurzrock's cohorts, respectively (figure 3C). Next, by comparing OS between patients with TMB-L $(\leq 13$ mut/Mb) and TMB-H (>13 mut/Mb) tumors, we found that patients with TMB-H tumors exhibited improved survival following ICB treatment in pooled tumors of high TMB (category I) and low TMB (category II) cancer types (figure 3D-E). However, only a small proportion (8.5\%$10.5 \%$ ) of patients with low TMB (category II) cancer types were TMB-H (>13 mut/Mb) (figure 3D-E, right plot). Thus, the clinical application of the $13 \mathrm{mut} / \mathrm{Mb}$ cut-off is relatively limited in low TMB (category II) cancer types.

\section{The difference in the significant TMB cut-offs for predicting favorable ICB treatment outcomes between pooled patients with high TMB (category I) and low TMB (category II) cancer types}

Next, we compared all the possible TMB cut-offs for predicting favorable ICB treatment outcomes between pooled patients with high TMB (category I) or low TMB (category II) cancer type. TMB-H was still associated with improved ICB treatment outcomes in the analyses of pooled tumors (figure 4A-B). However, it is worth noting that most of the TMB cut-offs $(>75 \%)$ were associated with significantly favorable OS following ICB treatment in high TMB (category I) cancer type (figure 4A). In contrast, a relatively small proportion of significantly favorable TMB cut-offs $(<30 \%)$ was found in low TMB (category II) cancer type (figure 4A). Furthermore, these findings were validated by analyzing the PFS in Kurzrock's cohort (figure 4B), indicating that TMB is a much more robust biomarker for predicting ICB response in high TMB (category I) cancer types than in those with low TMB (category II).

Moreover, pooled patients with high TMB (category I) and low TMB (category II) cancer types exhibited distinct distributions of significant TMB cut-offs for predicting favorable ICB treatment outcomes (figure 4C). The probability of significantly favorable TMB cut-offs peaked around 13 mut/Mb in high TMB (category I) tumors but not in low TMB (category II) tumors (figure 4C). Next, the above findings discovered using the Morris's cohort were successfully validated in Kurzrock's cohort independently (figure $4 \mathrm{D}$ ), indicating that the separation of diverse cancers into high TMB (category I) and low TMB (category II) cancer types is important for the clinical application of TMB in ICB treatment. Additionally, in both individual and pooled cancers, the clinical application of the 13 mut/Mb TMB cut-off was shown to be reliable in high TMB (category I) cancer types.

\section{TMB-H (>13 mut/Mb) predicts a better ICB response in high TMB (category I) cancer types but not in low TMB (category II) cancer types}

Since high TMB (category I) and low TMB (category II) cancer types showed distinct patterns in the association between TMB-H and ICB treatment outcomes, next, we investigated whether high TMB (category I) and low TMB (category II) cancer types were different in ICB response rates. The ICB responses were indicated by progressive disease, stable disease, partial response (PR) and complete response (CR). The efficacy of ICB treatment was evaluated using objective response rate (ORR), which was calculated by the percentage of PR and CR patients. ICB-treated patients with high TMB (category I) cancer types had a 24.4\% higher ORR than those with low TMB (category II) cancer types $(\mathrm{p}=0.054$; figure $5 \mathrm{~A})$. Additionally, patients with low TMB (category II) cancer types failed to achieve a 20\% ORR. Comparatively, patients with high TMB (category I) cancer types had $8.8 \%$ and $15.6 \%$ higher ICB response rates of $\mathrm{PR}$ and $\mathrm{CR}$, respectively (figure 5A).

Next, patients were stratified according to the TMB cutoff of 13 mut/Mb. Among the patients with low TMB (category II) cancer types, the TMB-H (>13 mut/Mb) group was not associated with an improved ICB response and even showed an $11.8 \%$ lower ORR than the TMB-L $(\leq 13$ mut/ $\mathrm{Mb}$ ) group (figure $5 \mathrm{~B}$, left plot). In contrast, among the patients with high TMB (category I) cancer types, the TMB-H (>13 mut/Mb) group had a $43.3 \%$ higher ORR than the TMB-L ( $\leq 13$ mut $/ \mathrm{Mb}$ ) group ( $<0.001$; figure $5 \mathrm{~B}$, right plot). The above findings showed that TMB-H ( $>13$ mut $/ \mathrm{Mb}$ ) was related to a better ICB response in high TMB (category I) cancer types but not in low TMB (category II) cancer types.

\section{TMB-H is associated with improved OS following ICB treatment in cancer of unknown primary (CUP) with a better- than-anticipated prognostic value}

In addition to the previously mentioned cancer types defined by their primary sites, a cancer type without a detectable primary tumor despite extensive clinical investigation, commonly known as cancer of unknown primary (CUP), raised our attention. Since CUP encompasses heterogeneous malignancies from unknown primary sites, the majority $(80 \%-85 \%)$ of CUP patients, who cannot be assigned to cancer type-specific therapies, are often treated with empirical chemotherapy and have a poor prognosis. ${ }^{18}$ A recent study revealed that CUP patients might potentially benefit from ICB treatment. ${ }^{19}$ However, there is still a lack of data on the identification of ICB-eligible CUP patients.

Herein, we explored whether TMB was predictive of OS in ICB-treated patients with CUP. TMB-H was associated with favorable ICB treatment outcomes in CUP patients (figure 6A). However, compared with high TMB (category I) cancer types in figure 1B, CUP had a relatively lower robustness of prognostic association. In univariate and multivariate analyses, only $8.9 \%$ and $16.8 \%$ significantly favorable TMB cut-offs were found, respectively (figure 6A), which indicated a limited robustness of using TMB to predict ICB response. The probability of significantly favorable TMB cut-offs in the CUP peaked at 12 mut/Mb (figure 6B). Next, CUP patients with TMB-H $(>12 \mathrm{mut} / \mathrm{Mb})$ tumors showed significantly improved OS following ICB than those with TMB-L ( $\leq 12$ mut/Mb) tumors $(\mathrm{p}=0.033$; figure $6 \mathrm{C})$. Thus, it seems applicable to 
A Morris' ICB cohort:
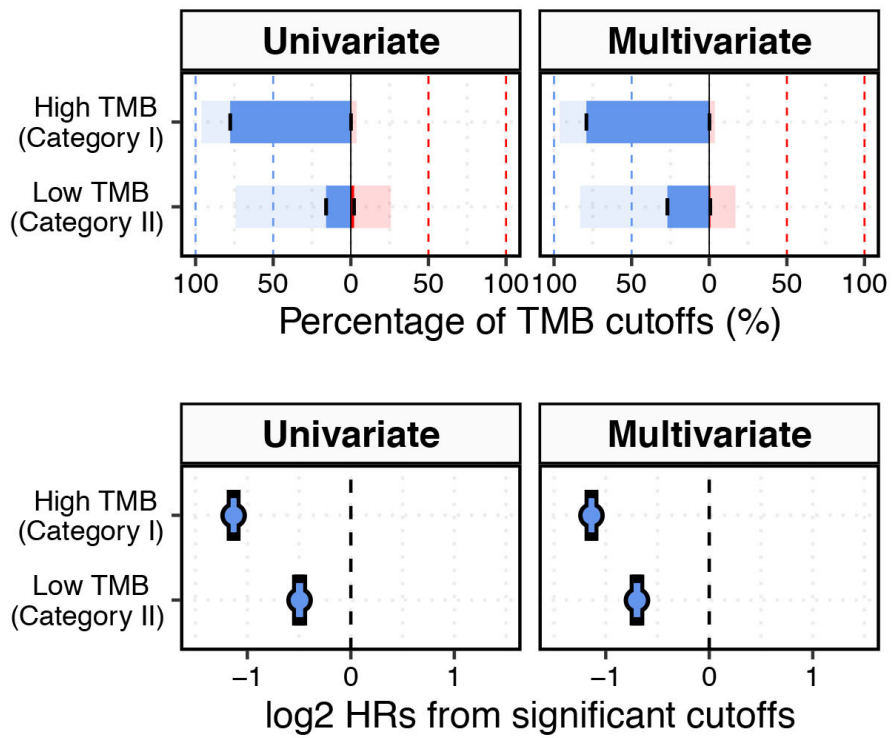

C Morris ICB cohort:

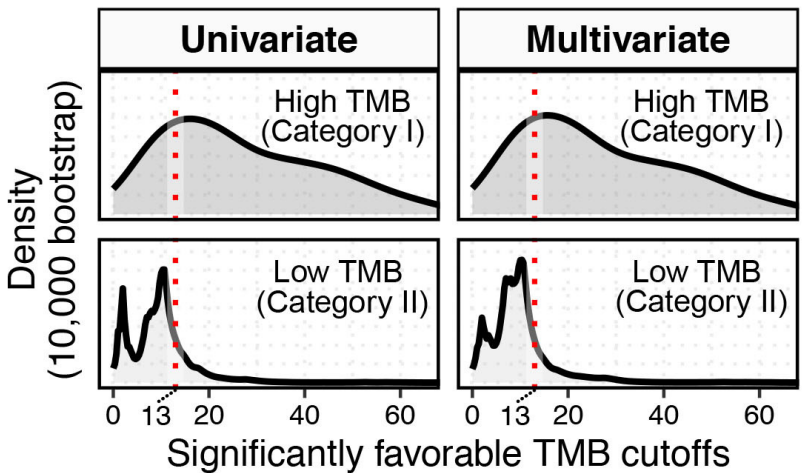

B Kurzrock's ICB cohort:
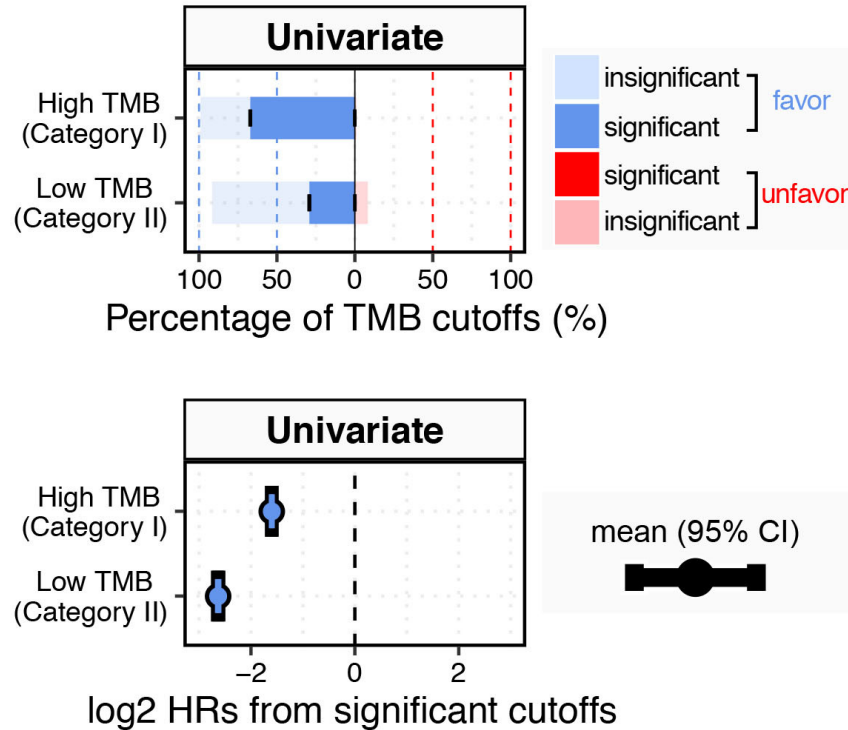

D Kurzrock's ICB cohort:

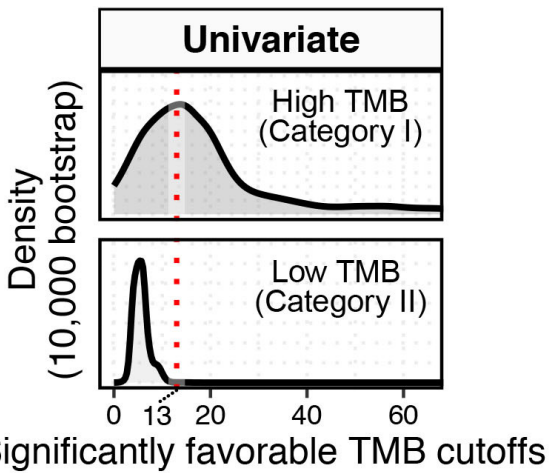

Figure 4 The difference in the significant TMB cut-offs for predicting favorable ICB treatment outcomes between pooled patients with high TMB (category I) and low TMB (category II) cancer types. (A-B) The prognostic relevance of TMB in pooled patients with high TMB (category I) and low TMB (category II) cancer types. Cox proportional hazards regression analyses were conducted to evaluate the OS in Morris's ICB cohort (A) and PFS in Kurzrock's ICB cohort (B). Multivariate analysis was performed with covariates of sex, age, drug class, and year of ICB start if applicable. Bar plots show the percentages of favorable and unfavorable TMB cut-offs, with error bars representing the SE calculated by 10,000 bootstrap replicates (upper plot). Dot plots show the average HR of significant TMB cut-offs, with error bars representing the mean $\pm 95 \% \mathrm{Cl}$ calculated by 10,000 bootstrap replicates (lower plot). Different colors correspond to favorable and unfavorable associations, with the color gradient showing prognostic significance. (C-D) The shift in the distributions of significant TMB cut-offs for predicting favorable ICB treatment outcomes in pooled patients with high TMB (category I) and low TMB (category II) cancer types. Density plots show the distributions of significantly favorable TMB cut-offs calculated by 10,000 bootstrap replicates from the Morris's (C) and Kurzrock's (D) ICB cohorts. The red dashed line indicates the TMB cut-off of 13 mut/Mb. ICB, immune checkpoint blockade; mut/Mb, mutations/megabase; OS, overall survival; PFS, progression-free survival; TMB, tumor mutation burden.

use TMB-H (>12 mut/Mb) as a predictive biomarker for predicting ICB response in CUP patients.

Next, we explored the relationship between the median TMB level in CUP patients and the robustness of the prognostic association between TMB-H and OS following ICB treatment. CUP had a median TMB level of $5.27 \mathrm{mut} / \mathrm{Mb}$, which was close to those of low TMB (category II) cancer types. Consistently, the robustness of its prognostic association between TMB-H and OS following ICB was also similar to low TMB (category II) cancer types (figure 6D-E). However, it is worth noting that CUP exhibited lower negative log2-HR compared with low TMB (category II) and even high TMB (category I) cancer types (figure 6F). Thus, the association between TMB-H and improved OS after ICB treatment was better than anticipated in CUP patients. Since CUP is a biologically enigmatic disease that encompasses heterogeneous cancers, this better-than-anticipated association in CUP patients still needs to be validated in further 


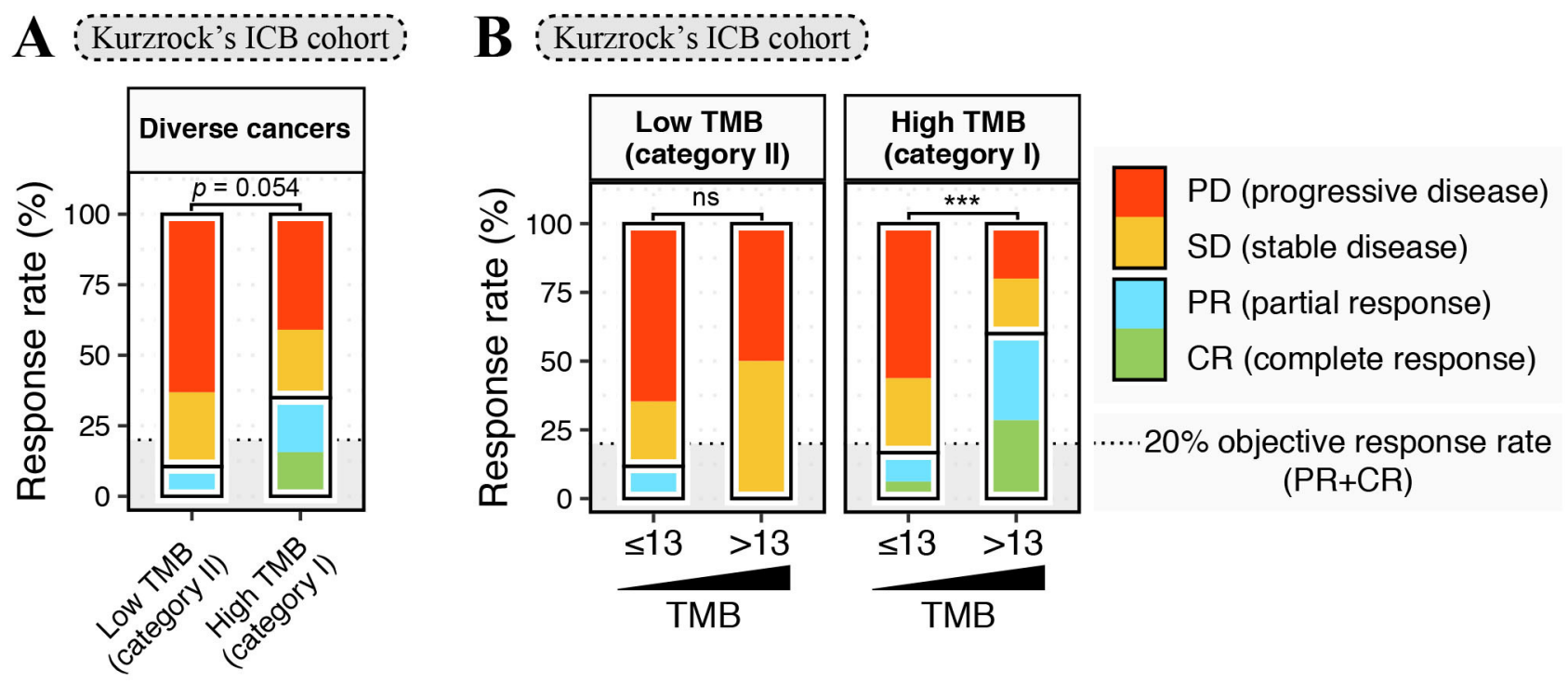

Figure 5 TMB-H (>13 mut/Mb) predicts a better ICB response in high TMB (category I) cancer types but not in low TMB (category II) cancer types. (A) ICB responses in high TMB (category I) and low TMB (category II) cancer types from Kurzrock's cohort. The stacked bar graph shows the percentage of different ICB responses, with color coding according to different ICB responses. (B) TMB for predicting ICB responses in high TMB (category I) and low TMB (category II) tumors. Patients were separated into subsets of tumors with TMB (mut/Mb) $\leq 13$ and $>13$. The results were considered statistically significant when $\left.\left.\mathrm{p}<0.05{ }^{*}\right), \mathrm{p}<0.01{ }^{(\star *}\right), \mathrm{p}<0.001\left(^{\star \star *}\right)$, and $\mathrm{p}<0.0001\left(^{\star \star \star *}\right)$ and insignificant when $\mathrm{p} \geq 0.05$ (ns) using the $\chi^{2}$ test with 10,000 replicates by Monte Carlo simulation. The black dashed line indicates a $20 \%$ objective response rate (ORR). ICB, immune checkpoint blockade; mut/Mb, mutations/megabase; CR, complete response; PD, progressive disease; PR, partial response; $\mathrm{SD}$, stable disease; TMB, tumor mutation burden; TMB-H, high tumor mutation burden.

studies. Nevertheless, as shown in figure 6D, CUP well fitted the correlation-TMB-H was more robustly associated with an improved ICB response in cancer types with more TMB, implying that this correlation might be extended to other cancer types.

\section{DISCUSSION}

Although the importance of TMB in predicting ICB response has increasingly been recognized, ${ }^{6} 20$ a robustness analysis of prognostic relevance using different TMB cutoffs has not been performed. In this study, using Morris's and Kurzrock's ICB cohorts, ${ }^{45}$ we characterized the landscape of TMB cut-offs for predicting improved ICB treatment outcomes across 10 cancer types. We observed that TMB-H was associated with favorable rather than unfavorable survival after ICB treatment in most cancer types. However, the prognostic associations were significantly affected by different selections of TMB cut-offs in low TMB (category II) cancer types, while high TMB (category I) cancer types were much less affected and showed remarkably robust associations between TMB-H and improved OS following ICB treatment.

To the best of our knowledge, this is the first study to report that TMB-H was more robustly associated with favorable ICB treatment outcomes in cancer types with more TMBs. Moreover, this correlation was even effective in a complex cancer type: CUP. Here, we propose a theory that this correlation can be used to predict whether TMB is a robust predictive biomarker in cancer types for which TMB data is available, but ICB treatment has not yet been investigated. Based on our theory, we can simply use the median TMB level of a cancer type to categorize it into a high TMB (category I) or low TMB (category II) cancer types. TMB-H is anticipated to be robustly predictive of improved survival in the high TMB (category I) cancer types but not in those with low TMB (category II).

This theory proposed in our study has practical and important utility considering the recent FDA approval of ICB treatment (anti-PD-1) in TMB-H patients. The FDA approval was based on the KEYNOTE-158 study, ${ }^{60}$ which lacked major cancer types for which anti-PD-1 treatment has not been approved, including prostate cancer and others. According to a previous study that found that prostate cancer has a low TMB level, ${ }^{16}$ we might estimate that TMB-H could not be a robust biomarker for predicting improved ICB response in prostate cancer.

Tumors with higher TMBs tend to be more responsive to ICB treatment, since they are thought to have more neoantigen loads available for $\mathrm{T}$ cells to respond to. Recently, it is reported that the presence of tumor-infiltrating $\mathrm{T}$ cells (TIL-T cells) is associated with ICB response. ${ }^{21}$ Thus, the interindividual differences in TIL-T cells might be an important piece of the puzzle for understanding ICB response. It is worth noting that TMB-H tumors exhibited better ICB response than TMB-L tumors in cancer types where $\mathrm{CD} 8^{+}$TIL-T cell levels positively correlated with neoantigen loads, including melanoma, colon, bladder, and lung cancers. ${ }^{8}$ However, not all CD $8^{+}$TIL-T cells recognize tumor antigens. A substantial subset of CD8 ${ }^{+}$TIL-T cells is bystander $\mathrm{T}$ cells that recognize a wide range of antigens 
A Cancer of unknown primary (CUP):
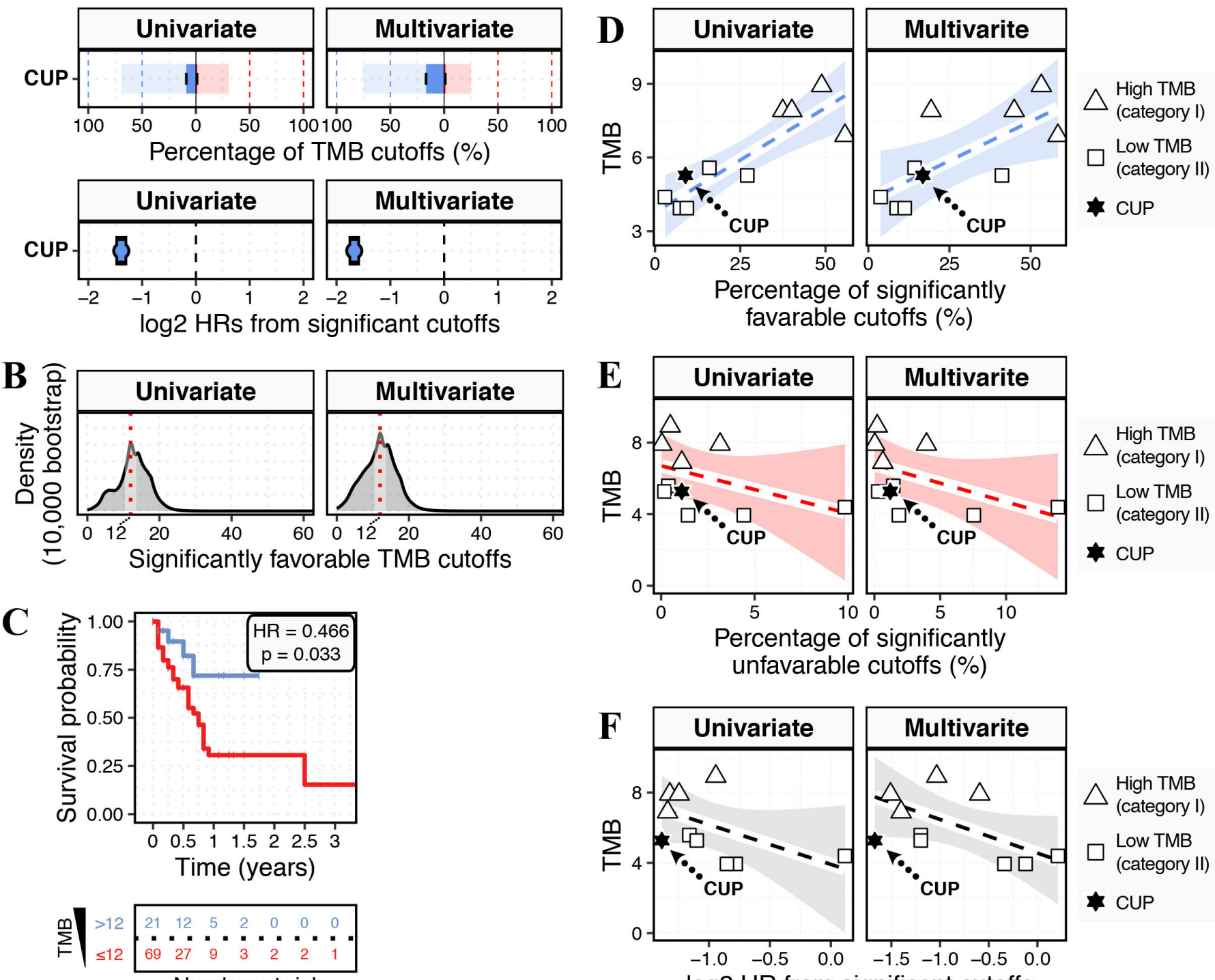

Number at risk

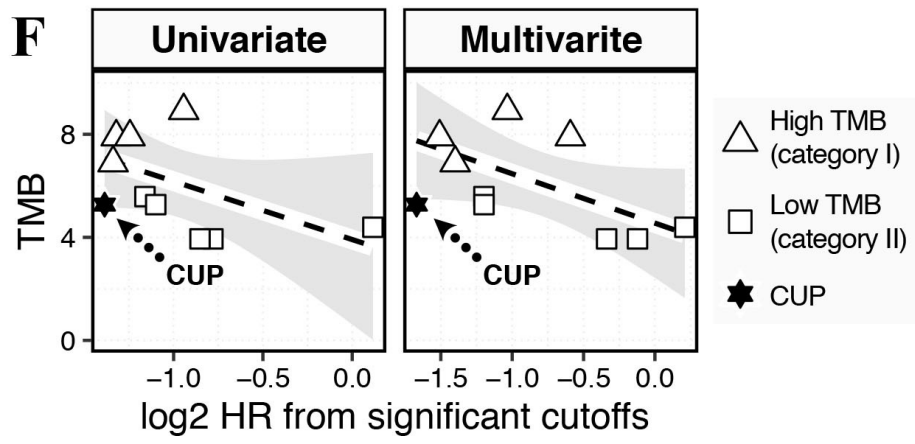

Figure 6 TMB-H is associated with improved OS following ICB treatment in cancer of unknown primary (CUP) with a better-than-anticipated prognostic value. (A) The prognostic relevance of TMB in CUP tumors from Morris's ICB cohort. Both univariate and multivariate Cox proportional hazards regression analyses were conducted to evaluate the OS of ICB-treated patients. Multivariate analysis was performed with covariates of sex, age, drug class, and year of ICB start. Bar plots show the percentages of favorable and unfavorable TMB cut-offs, with error bars representing the SE calculated by 10,000 bootstrap replicates (upper plot). Dot plots show the average HR of significant TMB cut-offs, with error bars representing the mean $\pm 95 \%$ $\mathrm{Cl}$ calculated by 10,000 bootstrap replicates (lower plot). Different colors correspond to favorable and unfavorable associations, with the color gradient showing the prognostic significance. (B) The distributions of significant TMB cut-offs for predicting the favorable ICB treatment outcomes of CUP tumors. Density plots show the distributions of significantly favorable TMB cut-offs calculated by 10,000 bootstrap replicates from Morris's ICB cohort. The red dashed line indicates the TMB cut-off of 12 mut/ Mb. (C) The Kaplan-Meier curves show the overall survival following ICB treatment in CUP patients with TMB-H and TMB-L tumors. The log-rank $p$ value and HR show the comparison between patients with TMB-L ( $\leq 12 \mathrm{mut} / \mathrm{Mb}$ ) and TMB-H (>12 mut/ $\mathrm{Mb})$. The bottom panel shows the number of patients at risk every half year. (D-F) The relationship of the median TMB values with the percentages of significantly favorable and unfavorable TMB cut-offs (D-E) and the average HRs of significant TMB cut-offs (F) in CUP, high TMB (category I), and low TMB (category II) cancer types. Dot shapes correspond to cancer types. The linear regression line with $95 \% \mathrm{Cl}$ was fitted using the data of high TMB (category I) and low TMB (category II) cancer types except for CUP. The dashed arrow shows the position of CUP. ICB, immune checkpoint blockade; mut/Mb, mutations/ megabase; OS, overall survival; TMB, tumor mutation burden; TMB-H, high tumor mutation burden; TMB-L, low tumor mutation burden.

unrelated to cancer. ${ }^{22}$ Thus, it is important to evaluate the quantity but also the quality of TIL-T cells. T cell antigen recognition relies on the $\mathrm{T}$ cell receptor (TCR) that directly interacts with its recognizable antigens. ${ }^{23}{ }^{24}$ Recently, based on T cell repertoire sequencing (TCR-seq), ${ }^{25}$ several bioinformatics approaches have been developed to identify and 
discriminate the antigenic specificity of the heterogeneous $\mathrm{T}$ cell clonotypes in diverse $\mathrm{T}$ cell repertoire. ${ }^{26}{ }^{27}$ Additionally, the TCR-seq-based immune signature has been reported to help identify cancer patients with an increased likelihood of responding to ICB treatment. ${ }^{28}{ }^{29}$ Therefore, it is apparent that both TMB and the neoantigen-related T cells in ICB responsiveness are important and need more attention, and further study is still required.

The clinical application of TMB in ICB treatment still faces several challenges, including differences in technology platforms, tissue and blood TMBs, and inadequate quantity and quality of tumor samples. ${ }^{15} 30$ Therefore, repeated and ongoing efforts have been made to overcome these limitations. A recent study demonstrated that the blood TMB measured by gene panel sequencing with optimized panel size and algorithm correlated well with tissue TMB calculated by whole-exome sequencing. ${ }^{31}$ Additionally, to overcome the problem of inadequate tissue volume and quality in tissue specimen-based TMB analysis, a pilot study has been conducted to show the successful use of cytological samples for TMB analysis. ${ }^{32}$

Besides the above-mentioned challenges, the reproducibility of TMB cut-off has not been thoroughly tested within and across cancer types, which has become a major limitation of its clinical application. ${ }^{15}$ This problem is especially important considering the recent FDA approval of ICB treatment in TMB-H (>10 mut/Mb) patients. ${ }^{78}$ In this study, we address this problem by exhaustively testing all possible TMB cut-offs in 10 cancer types, estimating the probability of how likely a given TMB cut-off would predict improved survival and using 10,000 randomly resampled cohorts to measure the reproducibility of each TMB cut-off. This analysis allowed us to find the optimal TMB cut-off within and across cancer types. Low TMB (category II) cancer types, for which the prognostic associations were sensitive to different TMB cut-off selection, showed markedly limited ranges of significantly favorable TMB cut-offs. In contrast, all the high TMB (category I) cancer types shared a wide range of TMB cut-offs for predicting improved ICB response, which allows us to discover an optimal and universal TMB cut-off of 13 mut/Mb. Moreover, the TMB cut-off of 13 mut/Mb was also optimally effective in the analyses of pooled tumors of high TMB (category I) cancer types. Our finding strongly suggests a robustly generalizable TMB cut-off of 13 mut/ $\mathrm{Mb}$ for high TMB (category I) cancer types, including melanoma, colorectal cancer, bladder cancer, and non-small cell lung cancer, thus, offering a reliable option for guiding the clinical application of ICB treatment.

TMB reflects the frequency of somatic mutations that give rise to neoantigens. Neoantigens increase the tumor immunogenicity by activating CD8 cytotoxic T cells (CTLs) that initiate tumor cell lysis. The ICB treatment depends on reinvigorating the cytolytic potential of CTLs to eliminate tumor cells. ${ }^{33}$ However, only part of the somatic mutations could lead to the formation of neoantigens, which needs to be presented by appropriate major histocompatibility complex (MHC) molecules on tumor cells, and occasionally recognized by specific TCR clonotypes from a highly diversified T cell repertoire. ${ }^{3}{ }^{34}$ Although it is difficult to determine whether a certain amount of somatic mutations are required to generate neoantigens that stimulate a functional immune response, it seems reasonable to assume that the more TMBs, the better success rate of ICB treatment. Therefore, the universal TMB cut-off identified in this study might reveal a general requirement to trigger the sequential cascade from somatic mutations to an effective antitumor immune response.

A limitation of our study is that only 10 cancer types were analyzed in this study. To fully measure this limitation, we estimated how many new cancer cases could attribute to the cancer types analyzed in our study. According to the cancer statistics of the USA in $2021,{ }^{35}$ the cancer types in our study present approximately more than half $(57.6 \%)$ of the estimated new invasive cancer cases (online supplemental figure S2). Moreover, to extend our findings to other cancer types, as discussed above, we proposed a theory that the findings in this study could be useful to speculate the relationship between TMB and ICB response in novel cancer types that were not analyzed in this study.

One of the important areas of future research in this field is whether patients with low TMB (category II) cancers could benefit from ICB treatment. ICB treatment has only modest activity in low TMB (category II) cancer types. For such cancer types, clinical trials have been conducted to study the combination therapies of ICB with targeted therapy, angiogenesis inhibitors, poly(ADPribose) polymerase inhibitors, radiotherapy, and chemotherapy. ${ }^{36}$ Among the previous combination immunotherapies, there is recently growing interest in adding ICB to the commonly used chemotherapies. It is reported that the combination therapy of ICB with chemotherapy that induces immunogenic cell death ${ }^{37}$ could boost the antitumor immune response elicited by ICB. ${ }^{38-40}$ Additionally, cytotoxic and mutagenic chemotherapies might convert TMB-L tumors to TMB-H status. ${ }^{41}{ }^{42}$ Moreover, ICB-chemotherapy combination therapy could increase the response rate and OS in low TMB cancers that are largely refractory to ICB monotherapy. ${ }^{36}{ }^{40}$ With more therapeutic approaches become available in the arsenal of cancer treatments, we will anticipate the future of further gains in the survival of patients with cancer.

Acknowledgements This project was supported by the National Natural Science Foundation of China (32100739) to MZ.

Contributors MZ conceived the project, developed the method, conducted data analysis, wrote the manuscript, and is the guarantor of this manuscript.

Disclaimer The funders had no role in the study design, data analysis, data interpretation, and writing of this manuscript. MZ supervised this project and is responsible for the overall content.

Competing interests None declared.

Patient and public involvement statement Not applicable.

Patient consent for publication Not applicable.

Ethics approval No human subjects were directly involved in this study. All the data used in this study were derived from existing deidentified biological samples and data from prior studies. Thus, ethical and patient consent were not required in this study. 
Provenance and peer review Not commissioned; externally peer reviewed.

Data availability statement Data are available on reasonable request.

Supplemental material This content has been supplied by the author(s). It has not been vetted by BMJ Publishing Group Limited (BMJ) and may not have been peer-reviewed. Any opinions or recommendations discussed are solely those of the author(s) and are not endorsed by BMJ. BMJ disclaims all liability and responsibility arising from any reliance placed on the content. Where the content includes any translated material, BMJ does not warrant the accuracy and reliability of the translations (including but not limited to local regulations, clinical guidelines, terminology, drug names and drug dosages), and is not responsible for any error and/or omissions arising from translation and adaptation or otherwise.

Open access This is an open access article distributed in accordance with the Creative Commons Attribution Non Commercial (CC BY-NC 4.0) license, which permits others to distribute, remix, adapt, build upon this work non-commercially, and license their derivative works on different terms, provided the original work is properly cited, appropriate credit is given, any changes made indicated, and the use is non-commercial. See http://creativecommons.org/licenses/by-nc/4.0/.

\section{ORCID iD}

Ming Zheng http://orcid.org/0000-0002-3651-7701

\section{REFERENCES}

1 Postow MA, Callahan MK, Wolchok JD. Immune checkpoint blockade in cancer therapy. JCO 2015;33:1974-82.

2 Topalian SL, Taube JM, Anders RA, et al. Mechanism-driven biomarkers to guide immune checkpoint blockade in cancer therapy. Nat Rev Cancer 2016;16:275-87.

3 Chan TA, Yarchoan M, Jaffee E, et al. Development of tumor mutation burden as an immunotherapy biomarker: utility for the oncology clinic. Ann Oncol 2019;30:44-56.

4 Goodman AM, Kato S, Bazhenova L, et al. Tumor mutational burden as an independent predictor of response to immunotherapy in diverse cancers. Mol Cancer Ther 2017;16:2598-608.

5 Samstein RM, Lee C-H, Shoushtari AN, et al. Tumor mutational load predicts survival after immunotherapy across multiple cancer types. Nat Genet 2019;51:202-6.

6 FDA. FDA approves pembrolizumab for adults and children with TMB-H solid tumors, 2020. Available: https://www.fda.gov/drugs/ drug-approvals-and-databases/fda-approves-pembrolizumabadults-and-children-tmb-h-solid-tumors

7 Prasad V, Addeo A. The FDA approval of pembrolizumab for patients with TMB >10 mut/Mb: was it a wise decision? No. Ann Oncol 2020;31:1112-4.

8 McGrail DJ, Pilié PG, Rashid NU, et al. High tumor mutation burden fails to predict immune checkpoint blockade response across all cancer types. Ann Oncol 2021;32:661-72.

9 DAaN P, Ajit J, Freeman TC, et al. Continuous biomarker assessment by exhaustive survival analysis. bioRxiv 2017;208660.

10 Cassetta L, Fragkogianni S, Sims AH, et al. Human tumor-associated macrophage and monocyte transcriptional landscapes reveal cancerspecific reprogramming, biomarkers, and therapeutic targets. Cancer Cell 2019;35:588-602.

11 Efron B. Nonparametric estimates of standard error: the jackknife, the bootstrap and other methods. Biometrika 1981;68:589-99.

12 Efron B. Better bootstrap confidence intervals. J Am Stat Assoc 1987;82:171-85.

13 Team RC. R: A language and environment for statistical computing: Vienna, Austria 2013.

14 Patefield WM. Algorithm AS 159: an efficient method of generating random $\mathrm{R} \times \mathrm{C}$ tables with given row and column Totals. App/ Stat 1981;30:91-7.

15 Addeo A, Banna GL, Weiss GJ. Tumor mutation burden-from hopes to doubts. JAMA Oncol 2019;5:934-5.

16 Zehir A, Benayed R, Shah RH, et al. Mutational landscape of metastatic cancer revealed from prospective clinical sequencing of 10,000 patients. Nat Med 2017;23:703-13.

17 Castle JC, Uduman M, Pabla S, et al. Mutation-derived neoantigens for cancer immunotherapy. Front Immunol 2019;10:10.

18 Rassy E, Pavlidis N. Progress in refining the clinical management of cancer of unknown primary in the molecular era. Nat Rev Clin Oncol 2020;17:541-54.
19 Haratani K, Hayashi H, Takahama T, et al. Clinical and immune profiling for cancer of unknown primary site. J Immunother Cancer 2019;7:251

20 Marabelle A, Fakih M, Lopez J, et al. Association of tumour mutational burden with outcomes in patients with advanced solid tumours treated with pembrolizumab: prospective biomarker analysis of the multicohort, open-label, phase 2 KEYNOTE-158 study. Lancet Oncol 2020;21:1353-65.

21 Jiang X, Dudzinski S, Beckermann KE, et al. MRI of tumor T cell infiltration in response to checkpoint inhibitor therapy. J Immunother Cancer 2020;8:e000328.

22 Simoni $\mathrm{Y}$, Becht $\mathrm{E}$, Fehlings $\mathrm{M}$, et al. Bystander CD8+ T cells are abundant and phenotypically distinct in human tumour infiltrates. Nature 2018;557:575-9

23 Davis MM, Boniface JJ, Reich Z, et al. Ligand recognition by alpha beta T cell receptors. Annu Rev Immunol 1998;16:523-44.

24 Garcia KC, Gapin L, Adams JJ, et al. A closer look at TCR germline recognition. Immunity 2012;36:887-8.

25 Freeman JD, Warren RL, Webb JR, et al. Profiling the T-cell receptor beta-chain repertoire by massively parallel sequencing. Genome Res 2009;19:1817-24.

26 Zheng $M$, Zhang $X$, Zhou Y, et al. TCR repertoire and CDR3 motif analyses depict the role of $\alpha \beta$ T cells in ankylosing spondylitis. EBioMedicine 2019;47:414-26.

27 Glanville J, Huang $\mathrm{H}$, Nau A, et al. Identifying specificity groups in the T cell receptor repertoire. Nature 2017:547:94-8.

28 Simnica D, Smits M, Willscher E, et al. Binder M: responsiveness to immune checkpoint inhibitors is associated with a peripheral blood Tcell signature in metastatic castration-resistant prostate cancer. JCO Precis Oncol 2020;4:1374-85.

29 Zhang $\mathrm{H}$, Liu L, Zhang J, et al. Investigation of antigen-specific T-cell receptor clusters in human cancers. Clin Cancer Res 2020;26:1359-71.

30 Galvano A, Gristina V, Malapelle U, et al. The prognostic impact of tumor mutational burden (TMB) in the first-line management of advanced non-oncogene addicted non-small-cell lung cancer (NSCLC): a systematic review and meta-analysis of randomized controlled trials. ESMO Open 2021;6:100124.

31 Wang Z, Duan J, Cai S, et al. Assessment of blood tumor mutational burden as a potential biomarker for immunotherapy in patients with non-small cell lung cancer with use of a next-generation sequencing cancer gene panel. JAMA Oncol 2019;5:696-702.

32 Pepe F, Pisapia P, Gristina V, et al. Tumor mutational burden on cytological samples: a pilot study. Cancer Cytopathol 2021;129:460-7.

33 Tumeh PC, Harview CL, Yearley JH, et al. PD-1 blockade induces responses by inhibiting adaptive immune resistance. Nature 2014;515:568-71.

34 Reuben A, Zhang J, Chiou S-H, et al. Comprehensive T cell repertoire characterization of non-small cell lung cancer. Nat Commun 2020;11:603

35 Siegel RL, Miller KD, Fuchs HE, et al. Cancer statistics, 2021. CA Cancer J Clin 2021;71:7-33.

36 Esteva FJ, Hubbard-Lucey VM, Tang J, et al. Immunotherapy and targeted therapy combinations in metastatic breast cancer. Lancet Oncol 2019;20:e175-86.

37 Pfirschke C, Engblom C, Rickelt S, et al. Immunogenic chemotherapy sensitizes tumors to checkpoint blockade therapy. Immunity 2016;44:343-54.

38 Passiglia F, Galvano A, Gristina V, et al. Is there any place for PD-1/ CTLA-4 inhibitors combination in the first-line treatment of advanced NSCLC? - a trial-level meta-analysis in PD-L1 selected subgroups. Trans/ Lung Cancer Res 2021;10:3106-19.

39 Wang Y, Xie W, Humeau J, et al. Autophagy induction by thiostrepton improves the efficacy of immunogenic chemotherapy. J Immunother Cancer 2020;8:e000462.

40 Janjigian YY, Shitara K, Moehler M, et al. First-line nivolumab plus chemotherapy versus chemotherapy alone for advanced gastric, gastro-oesophageal junction, and oesophageal adenocarcinoma (CheckMate 649): a randomised, open-label, phase 3 trial. Lancet 2021;398:27-40

41 Strickler JH, Hanks BA, Khasraw M. Tumor mutational burden as a predictor of immunotherapy response: is more always better? Clin Cancer Res 2021;27:1236-41.

42 Campbell BB, Light N, Fabrizio D, et al. Comprehensive analysis of hypermutation in human cancer. Cell 2017;171:1042-56. 